\title{
Hyperons Interaction Mediated by Strange Mesons and the Structure of Massive ProtoNeutron Stars
}

\author{
Xueling Mu Huanyu Jia* Xia Zhou \\ School of Physical Science and Technology, Southwest Jiaotong University, Chengdu \\ 610031, China \\ E-mail: hyjiadhome.swjtu.edu.cn
}

\begin{abstract}
Cosmic ray particles can be accelerated in the supernova. A protoneutron star is formed in a few seconds after the supernova explosion of a massive star. Neutron stars may be one of cosmic ray sources. Therefore, the study of structure of protoneutron stars is significant to investigating the origin and acceleration of cosmic rays. The hyperons interaction mediated by strange mesons and the structure of massive protoneutron stars are studied in the framework of the relativistic mean field theory. It is found that the strange mesons $\sigma^{*}$ and $\phi$ suppress the generation of hyperons in the protoneutron star matter. The temperature of the protoneutron star matter with $\sigma^{*}$ and $\phi$ mesons shows to be higher than that without them, and the equation of state is stiffer. The structure of the protoneutron star corresponding to the mass of PSR J0348+0432 are also presented.
\end{abstract}

Key Words:Cosmic ray Acceleration, Massive Protoneutron Star, Strange Mesons

35th International Cosmic Ray Conference - ICRC2017

10-20 July, 2017

Bexco, Busan, Korea

\footnotetext{
* Speaker.
} 


\section{Introduction}

Supernova remnants(SNRs) are generally considered as the sources of cosmic rays in the Galaxy and elsewhere [1], and shock acceleration in supernovae offers a plausible explanation for cosmic ray origin up to energy of $10^{14} \mathrm{eV}[2,3]$. SNRs exploding into a wind environment of the progenitor star and maximum nucleon energies of the order of $10^{15}-10^{6} \mathrm{eV}$ can be reached [4]. A cold neutron star is one kind of evolutionary outcome of a protoneutron $\operatorname{star}(\mathrm{PNS})$ which is formed in a few seconds after the supernova explosion of a massive $\operatorname{star}\left(M>8 M_{\odot}\right)$ [5]. Therefore, the study of structure of protoneutron stars is significant to investigating the origin and acceleration of cosmic rays.

In 2010, the massive neutron star PSR J1614-2230 was observed with $1.97 \pm 0.04 M_{\odot}$ [6], and in 2013, another massive neutron star PSR J0348+0432 of $2.01 \pm 0.04 M_{\odot}$ was observed [7]. So far, there are many papers have researched on the particle composition, the EOS, and the properties of these massive neutron stars matter [8-10]. Orsaria et al used a nonlocal extension of the SU(3) NambuCJona-Lasinio model and showed that massive neutron stars, such as PSR J1614-2230 with a gravitational mass of $1.97 \pm 0.04 M_{\odot}$, may contain mixtures of quarks and hadrons in their central regions [11].

The interaction of hyperons that may occur in neutron stars can mediated by scalar-meson $\left(\sigma^{*}\right)$ and vector-meson $(\phi)$ introduced by Schaffner et al [12-14]. Basing on RMF approach, Oertel et al studied neutron star matter including hyperonic degrees of freedom and the hyperon-hyperon interaction is described through the $\sigma^{*}$ and $\phi$ mesons [15]. Bednarek et al constructed a model of the hyperon cores of neutron stars that allows for the existence of neutron star of $2 \mathrm{M}_{\odot}$ and they also introduced strange mesons fields coupled only to hyperons [16]. Gomes et al developed a new class of EOSs that allows for the presence of hyperons, and the strange mesons play an important role in the description of hyperon interactions [17].

\section{Relativistic mean field theory at definite entropy}

The RMF theory is an effective field theory of hadron interaction [18]. The interaction of baryons in neutron stars is achieved by exchanging $\sigma, \omega, \rho$ mesons, $\sigma$ provides the medium-range attractive interactions, $\omega$ provides short range repulsive interactions, $\rho$ describes the difference between neutron and proton. The interacting of hyperons that may occur in neutron stars mediated by the strange mesons $\sigma^{*}$ and $\phi$ introduced by Schaffner et al [12-14].

The Lagrangian density of the neutron star matter containing strange mesons $\sigma^{*}$ and $\phi$ is $[19,20]:$

$$
\begin{aligned}
\ell= & \sum_{B} \bar{\psi}_{B}\left(i \gamma_{\mu} \partial^{\mu}-m_{B}+g_{\sigma B} \sigma-g_{\omega B} \gamma_{\mu} \omega^{\mu}-\frac{1}{2} g_{\rho B} \gamma_{\mu} \tau \cdot \rho^{\mu}\right) \psi_{B} \\
& +\frac{1}{2}\left(\partial_{\mu} \sigma \partial^{\mu} \sigma-m_{\sigma}^{2} \sigma^{2}\right)-\frac{1}{3} g_{2} \sigma^{3}-\frac{1}{4} g_{3} \sigma^{4}-\frac{1}{4} \omega_{\mu \nu} \omega^{\mu \nu}+\frac{1}{2} m_{\omega}^{2} \omega_{\mu} \omega^{\mu} \\
& -\frac{1}{4} \rho_{\mu \nu} \cdot \rho^{\mu v}+\frac{1}{2} m_{\rho}^{2} \rho_{\mu} \cdot \rho^{\mu}+\sum_{\lambda=e, \mu} \bar{\psi}_{\lambda}\left(i \gamma_{\mu} \partial^{\mu}-m_{\lambda}\right) \psi_{\lambda}+\ell^{Y Y}
\end{aligned}
$$

where $\psi_{B}$ is the field operator of baryon $\mathrm{B}(\mathrm{n}, \mathrm{p}, \Lambda, \Sigma, \Xi)$ and the corresponding bare mass is $m_{B}$. $\sigma, \omega, \rho$ represent the field of scalar meson $\sigma$, vector meson $\omega$, and $\rho$ meson, respectively, and 
$m_{\sigma}, m_{\omega}, m_{\rho}$ are their corresponding masses. $g_{\sigma B}, g_{\omega B}, g_{\rho B}$ denote the coupling strength between baryon B and $\sigma, \omega, \rho$ mesons, $g_{2}, g_{3}$ are the self-interaction of $\sigma$ meson. The term $\ell^{Y Y}$ stands for the contribution of the $\sigma^{*}$ meson and $\phi$ meson and reads [13]

$$
\begin{aligned}
\ell^{Y Y}= & \sum_{B} g_{\sigma^{*} B} \bar{\psi}_{B} \psi_{B} \sigma^{*}-\sum_{B} g_{\phi B} \bar{\psi}_{B} \gamma_{\mu} \psi_{B} \phi^{\mu}+ \\
& \frac{1}{2}\left(\partial_{\mu} \sigma^{*} \partial^{\mu} \sigma^{*}-m_{\sigma^{*}}^{2} \sigma^{* 2}\right)-\frac{1}{4} S_{\mu v} S^{\mu v}+\frac{1}{2} m_{\phi}^{2} \phi_{\mu} \phi^{\mu}
\end{aligned}
$$

here, $S_{\mu \nu}=\partial_{\mu} \varphi_{v}-\partial_{\nu} \varphi_{\mu}, \sigma^{*}$ and $\phi$ represent the field of scalar-meson $\sigma^{*}$ and vector-meson $\phi$, respectively.

The partition function of a hot matter system is [21]:

$$
\begin{aligned}
\ln Z= & \frac{V}{T}<\ell>+\sum_{B} \frac{2 J_{B}+1}{2 \pi^{2}} \int_{0}^{\infty} \kappa^{2} d \kappa \ln \left(1+e^{-\left(\varepsilon_{B}(\kappa)-\mu_{B}\right) / T}\right)+ \\
& +\sum_{\lambda=e, \mu} \frac{1}{\pi^{2}} \int_{0}^{\infty} \kappa^{2} d \kappa \ln \left(1+e^{-\left(\varepsilon_{\lambda}-\mu_{\lambda}\right) / T}\right)
\end{aligned}
$$

where $\mathrm{V}$ is the volume of the system.

The energy density and pressure of the system are related to the partition function as follows:

$$
\varepsilon=\frac{T^{2}}{V} \frac{\partial}{\partial T} \ln Z, P=\frac{T}{V} \ln Z
$$

Therefore, the energy density and pressure of hot neutron star matter system are:

$$
\begin{aligned}
\varepsilon= & \frac{1}{2} m_{\sigma}^{2} \sigma^{2}+\frac{1}{3} g_{2} \sigma^{3}+\frac{1}{4} g_{3} \sigma^{4}+\frac{1}{2} m_{\omega}^{2} \omega_{0}^{2}+\frac{1}{2} m_{\rho}^{2} \rho_{03}^{2}+\frac{1}{2} m_{\sigma^{*}}^{2} \sigma^{* 2}+\frac{1}{2} m_{\phi}^{2} \phi_{0}^{2} \\
& \sum_{B} \frac{2 J_{B}+1}{2 \pi^{2}} \int_{0}^{\infty} \sqrt{\kappa^{2}+\left(m_{B}-g_{\sigma B} \sigma-g_{\sigma^{*} B} \sigma^{*}\right)^{2}}\left(\exp \left[\left(\varepsilon_{B}(\kappa)-\mu_{B}\right) / T+1\right)^{-1} \kappa^{2} d \kappa\right. \\
& +\sum_{\lambda=e, \mu} \frac{1}{\pi^{2}} \int_{0}^{\infty} \sqrt{\kappa^{2}+m_{\lambda}^{2}} n_{\lambda}(\kappa) \kappa^{2} d \kappa \\
P=- & \frac{1}{2} m_{\sigma}^{2} \sigma^{2}-\frac{1}{3} g_{2} \sigma^{3}-\frac{1}{4} g_{3} \sigma^{4}+\frac{1}{2} m_{\omega}^{2} \omega_{0}^{2}+\frac{1}{2} m_{\rho}^{2} \rho_{03}^{2}-\frac{1}{2} m_{\sigma^{*}}^{2} \sigma^{* 2}+\frac{1}{2} m_{\phi}^{2} \phi_{0}^{2} \\
+ & \frac{1}{3} \sum_{B} \frac{2 J_{B}+1}{2 \pi^{2}} \int_{0}^{\infty} \frac{\kappa^{2}}{\sqrt{\kappa^{2}+\left(m_{B}-g_{\sigma B} \sigma-g_{\sigma^{*} B} \sigma^{*}\right)^{2}}}\left(\exp \left[\varepsilon_{B}(\kappa)-\mu_{B}\right) / T+1\right)^{-1} \kappa^{2} d \kappa \\
+ & \frac{1}{3} \sum_{\lambda=e, \mu} \frac{1}{\pi^{2}} \int_{0}^{\infty} \frac{\kappa^{4}}{\sqrt{\kappa^{2}+m_{\lambda}^{2}}} n_{\lambda}(\kappa) d \kappa
\end{aligned}
$$

The total entropy per baryon of PNS matter system is expressed as

$$
S=\left(P+\varepsilon-\sum_{B} \rho_{B} \mu_{B}\right) / T \rho
$$

where $\rho=\sum_{B} \rho_{B}$ is the total baryon number. 


\section{Coupling parameters}

There have been many sets of nucleon coupling parameters. Here, we choose the nucleon coupling parameter set GL85 [22] to calculate the properties of massive neutron stars. For the hyperon $\mathrm{Y}(\Lambda, \Sigma, \Xi)$, we introduce the ratios of the hyperon coupling constants to the nucleon coupling constants

$$
x_{\sigma Y}=\frac{g_{\sigma Y}}{g_{\sigma N}}, x_{\omega Y}=\frac{g_{\omega Y}}{g_{\omega N}}, x_{\rho Y}=\frac{g_{\rho Y}}{g_{\rho N}}
$$

where $\mathrm{N}$ represents the nucleons (neutron and proton). The vector meson coupling parameters of $\rho$ are determined from $\mathrm{SU}(6)$ symmetry [23,24]

$$
2 g_{\rho N}=g_{\rho \Sigma}, g_{\rho N}=g_{\rho \Xi}, g_{\rho \Lambda}=0
$$

The coupling parameters of hyperon $\mathrm{Y}$ with the scalar meson $\sigma$ and vector meson $\omega$ satisfy the following relation $[10,25]$

$$
U_{Y}^{N}=x_{\omega Y} V-x_{\sigma Y} S
$$

Here, $V=\left(g_{\omega N} / m_{\omega}\right)^{2} \cdot \rho_{0}, S=m-m^{*}$, are the scalar potential and vector potential of the nucleon at saturation density $\rho_{0}$, respectively. $U_{Y}^{N}$ is the hypernuclear potential depth in saturation nuclear matter. In this calculation,we take $U_{\Lambda}^{N}=-30 \mathrm{MeV}, U_{\Sigma}^{N}=+30 \mathrm{MeV}, U_{\Xi}^{N}=-28 \mathrm{MeV}$ [26-28], and fix $x_{\sigma \Lambda}=0.8, x_{\sigma \Sigma}=0.4, x_{\sigma \Xi}=0.8$ according to the paper [29], then the coupling parameters of vector meson $\omega$ can be obtained as $x_{\omega \Lambda}=0.9320, x_{\omega \Sigma}=0.7597, x_{\omega \Xi}=0.9450$ respectively.

The coupling constants of strange scalar meson $\sigma^{*}$ and strange vector meson $\phi$ are also determined by SU(6) symmetry:

$$
\left\{\begin{array}{l}
2 g_{\sigma^{*} \Lambda}=2 g_{\sigma^{*} \Sigma}=g_{\sigma^{*} \Xi}=\frac{2 \sqrt{2}}{3} g_{\sigma N} \\
2 g_{\phi \Lambda}=2 g_{\phi \Sigma}=g_{\phi \Xi}=\frac{2 \sqrt{2}}{3} g_{\omega N}
\end{array}\right.
$$

With the EoS calculated with the above coupling parameters, the mass and radius of the neutron star can be obtained by solving the Oppenheimer-Volkoff (OV)equations [30]

$$
\left\{\begin{array}{l}
\frac{d p}{d r}=\frac{[p(r)+\varepsilon(r)]\left[M(r)+4 \pi r^{3} p(r)\right]}{r[r-2 M(r)]} \\
M(r)=4 \pi \int_{0}^{r} \varepsilon(r) r^{2} d r
\end{array}\right.
$$

In the $\mathrm{OV}$ equation, $\mathrm{r}$ is the radial distance from the center of the star, $P(r)$ is the pressure, $\varepsilon(r)$ is the energy density and $M(r)$ is the mass.

The relationship between the mass and the radius of the neutron star with or without considering the strange mesons are shown in Fig.1. Without the strange mesons, the maximum mass and the corresponding radius are $2.0559 M_{\odot}$ and $11.899 \mathrm{~km}$. While including the $\sigma^{*}$ and $\phi$ mesons, the maximum mass and the corresponding radius are $2.0645 M_{\odot}, 11.839 \mathrm{~km}$. It can be seen that the maximum mass of cold neutron stars with the strange mesons exceeds $2 M_{\odot}$. Therefore, the parameter set GL85 can be used to describe the $2 M_{\odot}$ massive neutron star. 


\section{Results and discussion}

The above coupling parameters model is extrapolated to study the properties of massive PNSs with per baryon entropy $S=1$. The effects of strange mesons on the properties of the massive PNSs is focused on. Firstly, we calculate various meson fields and chemical potentials of PNS matter.
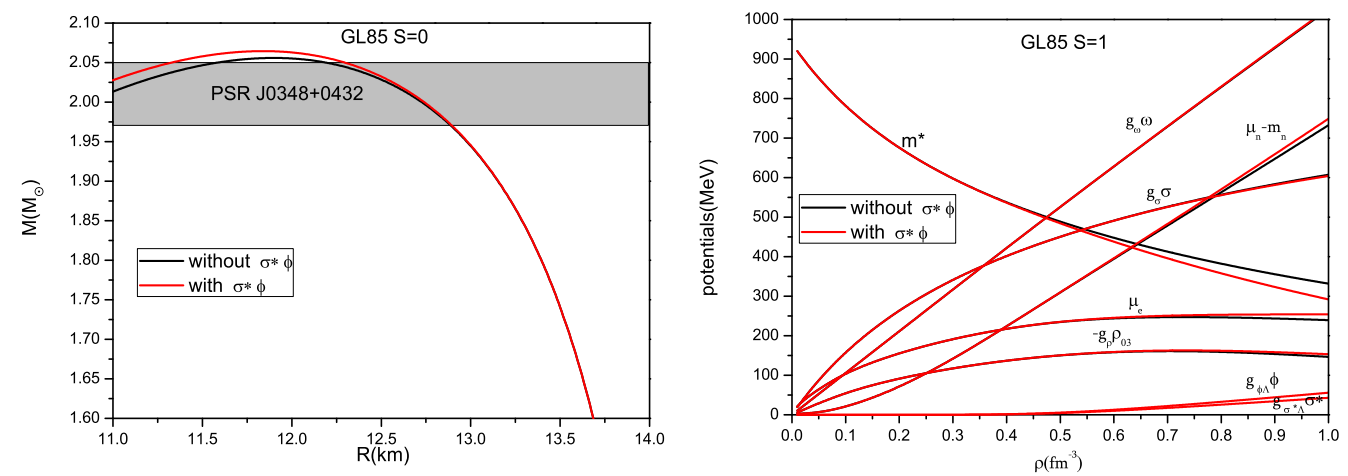

Figure 1: The mass-radius relationship of cold Figure 2: The field strengths, chemical potenneutron stars. The shaded area corresponds to tials and nucleon effective mass as functions of the mass of PSR J0348+0432. baryon density.

Fig.2 displays the field strengths, chemical potentials and nucleon effective mass as functions of baryon density of the PNSs matter. With rising total baryon density, the field strengths of the scalar field $\sigma$ and vector field $\omega_{0}$ and the neutron chemical potential $\mu_{n}$ increase rapidly, while the field strengths of isospin-vector $\rho_{03}$ and the electron chemical potentials $\mu_{e}$ are relatively small and change gently. The repulsive potential of $\phi$ meson is larger than the attractive potential of $\sigma^{*}$ meson. Compared with those without considering strange mesons, the meson field of $\rho_{03}$ and chemical potentials of $\mu_{n}$ and $\mu_{e}$ are greater than those without the strange mesons at high density. As the baryon number density increases, the effective mass of nucleon descend obviously.

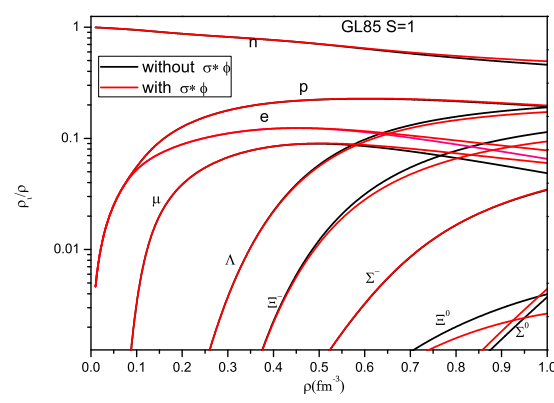

(a)

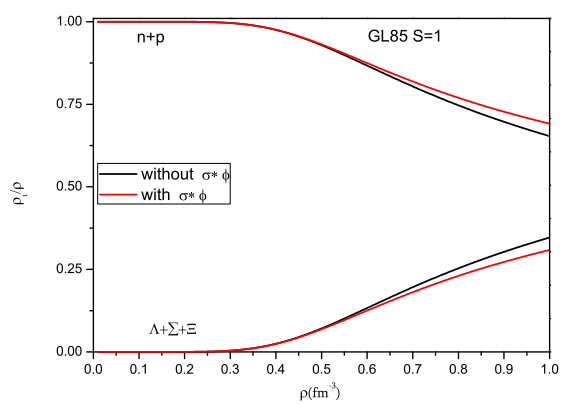

(b)

Figure 3: The particle number distribution.(a) Relative populations of particles as a function of baryon density.(b)Relative populations of nucleons and hyperons as a function of baryon density. 
The particles number distribution of the PNSs are given in Fig.3. Fig.3(a) presents the relative populations of different kinds of particles as a function of baryon density. At low density, the PNS matter mainly consists of neutrons, and a small amount of the same number of protons and electrons to maintain electric charge neutrality; As the density increases, muons and the hyperons $\Lambda, \Xi^{-}, \Sigma^{-}, \Xi^{0}, \Sigma^{0}$ successively appeare. The appearance of a particle here means that the relative proportion of the particle begins to outnumber $10^{-3}$. Fig.3(b) presents the relative populations of nucleons(protons and neutrons) and all hyperons as a function of baryon number density. It can be seen that when the baryon density is larger than $0.3 \mathrm{fm}^{-3}$, the proportion of nucleons begins to fall from 1, while the hyperons begin to appear and the sum of their relative number density increases rapidly. That is to say, the strange mesons suppress the appearance of the hyperons in the PNSs. We calculate the energy density and the pressure of the protoneutron star matter, and the results
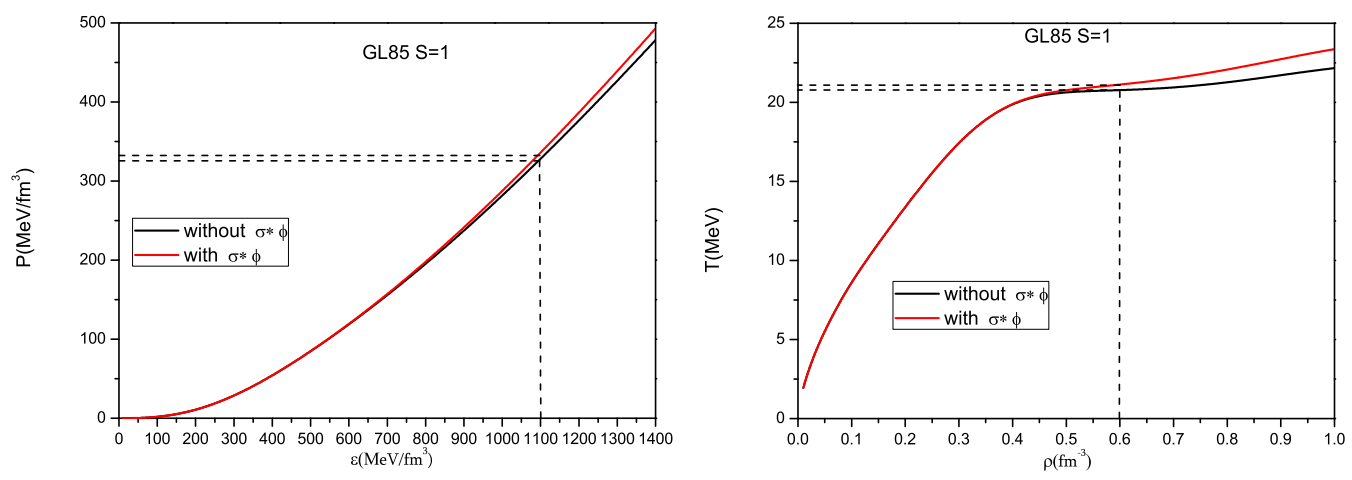

Figure 4: The equations of state. The dashed Figure 5: The temperature. The dashed line line indicates the pressure corresponding to the indicate the temperatures corresponding to the energy density $\varepsilon=1100 \mathrm{MeV} / \mathrm{fm}^{-3}$. density $\rho=0.6 \mathrm{fm}^{-3}$.

are shown in Fig.4. It can be seen that the EoS with the $\sigma^{*}$ meson and $\phi$ meson is stiffer than that without the $\sigma^{*}$ and $\phi$ mesons. For example, at the energy density $\varepsilon=1100 \mathrm{MeV} / \mathrm{fm}^{-3}$, the pressure changes from $328 \mathrm{MeV} / \mathrm{fm}^{-3}$ without the $\sigma^{*}$ and $\phi$ mesons to $336 \mathrm{MeV} / \mathrm{fm}^{-3}$ including the $\sigma^{*}$ meson and $\phi$ meson. This is due to the softening effects of hyperons in the neutron star matter on the EoS.

Further, the internal temperature of PNS is calculated and the results are illustrated in Fig.5. As can be seen from Figure 5, in the low-density region, as the density increases, the temperature increases rapidly. When the baryon density becomes larger than $0.4 \mathrm{fm}^{-3}$, the increase in temperature slows down as the baryon number density increases. In this process, the $\sigma^{*}$ meson and $\phi$ meson make the temperature higher. For instance, at the density $\rho=0.6 \mathrm{fm}^{-3}$, the temperature is $20.772 \mathrm{MeV}$ without considering the $\sigma^{*}$ and $\phi$ mesons, while the temperature is $21.125 \mathrm{MeV}$ when the strange mesons are taken into account.

On the basis of the above EoS, the mass and radius of the PNS are calculated by solving the OV equation, the results are shown in Fig.6. The shaded portion corresponds to the mass of the neutron star PSR $0348+0432$. The maximum mass of PNS is $2.0654 M_{\odot}$ and its corresponding 


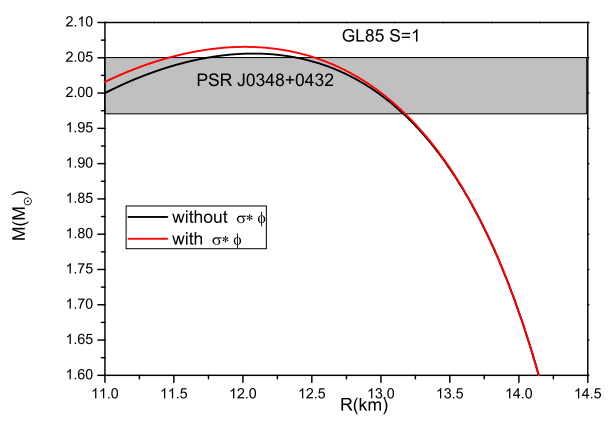

Figure 6: The mass-radius relation of massive protonuetron star. The shaded portion corresponds to the mass of the neutron star PSR 0348+0432.

Table 1: he properties of neutron star PSR 0348+0432 and protoneutron star of equal mass

\begin{tabular}{ccccccc}
\hline \hline & $M$ & $\mathrm{R}$ & $\rho_{C}$ & $\varepsilon_{C}$ & $P_{C}$ & $T_{C}$ \\
$\left(M_{\odot}\right)$ & $(\mathrm{km})$ & $\left(\mathrm{fm}^{-3}\right)$ & $\left(10^{15} \mathrm{~g} / \mathrm{cm}\right)$ & $\left(10^{35} \mathrm{dyne} / \mathrm{cm}^{2}\right)$ & $(\mathrm{MeV})$ \\
\hline$S=0$ without $\sigma^{*} \phi$ & $2.01_{-0.04}^{+0.04}$ & $12.653_{+0.235}^{-0.465}$ & $0.698_{-0.066}^{+0.137}$ & $1.425_{-0.165}^{+0.363}$ & $3.146_{-0.585}^{+1.407}$ & 0 \\
$S=0$ with $\sigma^{*} \phi$ & $2.01_{-0.04}^{+0.04}$ & $12.672_{+0.378}^{+0.223}$ & $0.689_{-0.062}^{+0.06}$ & $1.403_{-0.153}^{+0.279}$ & $3.098_{-0.553}^{+1.107}$ & 0 \\
$S=1$ without $\sigma^{*} \phi$ & $2.01_{-0.04}^{+0.04}$ & $12.903_{+0.512}^{+0.258}$ & $0.685_{-0.065}^{+0.137}$ & $1.405_{-0.161}^{+0.364}$ & $3.051_{-0.569}^{+1.407}$ & $20.908_{-0.111}^{+0.451}$ \\
$S=1$ with $\sigma^{*} \phi$ & $2.01_{-0.04}^{+0.04}$ & $12.932_{+0.409}^{-0.245}$ & $0.674_{-0.060}^{+0.105}$ & $1.377_{-0.148}^{+0.275}$ & $2.988_{-0.538}^{+1.075}$ & $21.410_{-0.236}^{+0.537}$ \\
\hline M,R, $\rho_{C}, \varepsilon_{C}, P_{C}, T_{C}$ denote mass, radius, center density, central energy density, center pressure &
\end{tabular}

radius is $12.014 \mathrm{~km}$ when $\sigma^{*}$ meson and $\phi$ meson are considered. While the maximum mass of PNS and the corresponding radius are $2.056 M_{\odot}$ and $12.077 \mathrm{~km}$ without $\sigma^{*}$ meson and $\phi$ meson.

In Table 1, the properties of PSR J0348 + 0432 and the PNS corresponding to the mass of PSR $\mathrm{J} 0348+0432$ are presented. Compared with the massive cold neutron star, the radius of the PNS becomes larger.

\section{Summary}

In this paper, by the appropriate hyperon coupling parameters in the parameter set GL85 and including the $\sigma^{*}$ meson and $\phi$ meson interaction, the maximum mass of the cold neutron star are calculated to be $2.0645 M_{\odot}$, and the corresponding radius is $11.899 \mathrm{~km}$.

The results reveal that the repulsive potential of $\phi$ meson is larger than the attractive potential of $\sigma^{*}$ meson. The population of the hyperon with the strange mesons is smaller than that without the strange mesons. The temperature of PNS matter with the $\sigma^{*}$ meson and $\phi$ meson is higher than that without strange mesons. By comparison, the results also show that the EOS of PNS is stiffer when the $\sigma^{*}$ and $\phi$ mesons are taken into account, and the maximum mass of the PNS is larger.

The properties of PSR J0348 + 0432 and the PNS whose mass is same as PSR J0348 + 0432 are also presented. The radius of the massive PNS becomes larger than that of cold neutron star. 
These results is significant to investigating the evolution of neutron star and the acceleration and origin of cosmic rays.

\section{References}

[1] Axford, W.I., 1981, Proc. 17th Internat. Cosmic Ray Conference, 12, 155

[2] Blandford R., 1987, Eichler D., Physics Reports, 154(1): 1-75

[3] Axford, W.I., 1994, Astrophys. J. Suppl. 90, 937

[4] Voelk H. J., 1988, The Astrophysical Journal, 333: L65-L68

[5] Bethe,H. A., 1990, Rev.Mod.Phys., 62,801

[6] Demorest, P. B., Pennucci, T., Ransom, S. M., et al. 2010, nature, 467, 1081

[7] Antoniadis, J., Freire, P. C. C, Wex, N., et al. 2013, Science, 340, 1233232

[8] Haensel, P., \& Zdunik, J. L. 2016, Journal of Physics: Conference Series. IOP Publishing, 665, 012061

[9] Orsaria, M., Friedman, H., Weber, F., et al. 2014, PRC, 89, 015806

[10] Weissenborn, S., Chatterjee, D., Schaffner-Bielich, J., et al. 2012, Nuclear Physics A, 881, 62

[11] Orsaria, M., Friedman, H., Weber, F., et al. 2013, PRD, 87, 023001

[12] Schaffner, J., Dover, C. B., Gal, A., et al. 1993, PRL, 71, 1328

[13] Schaffner, J., Dover, C. B., Gal, A., et al. 1994, Ann. Phys., 235, 35

[14] Glendenning, N. K., 1985, APJ, 293, 470

[15] Oertel, M., ProvidÃłncia, C., Gulminelli, F., et al. 2015, J. Phys. G.:Nuclear and Particle Physics, 42, 075202

[16] Bednarek, I., Haensel, P., Chung,J. L., 2012, Astron. and Astrophys, 543, A157

[17] Gomes, R. O., Dexheimer, V., Schramm, S., et al. 2015, APJ, 808, 8

[18] Glendenning, N. K., 1982, Phys.Lett.B, 114, 392

[19] Glendenning, N. K., 1987, Nuclear Physics A, 469, 600

[20] Zhao, X. F., Wang, S. J. Zhang, H., et al. 2007, HEP \& NP, 31, 345

[21] Prakash M., Bombaci I., Prakash M., et al. 1997. Physics Reports, 280(1): 1-77

[22] Glendenning, N. K., 1985, APJ, 293, 470

[23] S., Hanauske, M., Zakout, I., et al. 1999, PRC, 60, 015802

[24] Schaffner, J., \& Mishustin, N., 1996, PRC, 53, 1416

[25] Muller, H., 1999, PRC, 59, 1405

[26] Kohno, M., Fujiwara, Y., Watanabe, Y., et al. 2006, PRC, 74, 064613

[27] Friedman, E., \& Gal,A., 2007, Phys. Rep., 452, 89

[28] Batty, C. J., Friedman, E., Gal, A., 1997, Phys. Rep., 287, 385

[29] Zhao, X. F., 2014, Eur. Phys. J. A, 50, 80

[30] Oppenheimer, J. R., \& Volkoff G. M., 1939, Phys. Rev., 55, 374 